Check for updates

Cite this: RSC Adv., 2018, 8, 13353

Received 22nd January 2018

Accepted 6th April 2018

DOI: $10.1039 / \mathrm{c} 8 \mathrm{ra00637g}$

rsc.li/rsc-advances

\section{Preparation and characterization of an amphiphilic polyamide nanofiltration membrane with improved antifouling properties by two-step surface modification method}

\author{
Huimin Ruan, ${ }^{\mathrm{a}}$ Bin Li ${ }^{a}$ Jianbing Ji, ${ }^{\mathrm{b}}$ Arcadio Sotto, ${ }^{\mathrm{c}}$ Bart Van der Bruggen, ${ }^{\mathrm{d}}$ \\ Jiangnan Shen (DD ${ }^{* a}$ and Congjie Gao ${ }^{a}$
}

Membrane fouling is an urgent problem needing to be solved for practical application of nanofiltration membranes. In this study, an amphiphilic nanofiltration membrane with hydrophilic domains as well as low surface energy domains was developed, to integrate a fouling-resistant defense mechanism and a fouling-release defense mechanism. A simple and effective two-step surface modification of a polyamide NF membrane was applied. Firstly, triethanolamine (TEOA) with abundant hydrophilic functional groups was grafted to the membrane surface via reacting with the residual acyl chloride group of the nanofiltration membrane, making the nanofiltration membranes more hydrophilic; secondly, the $1 H, 1 H, 2 H, 2 H$-perfluorodecyltrichlorosilane (PFTS), well-known as a low surface energy material, was covalently grafted on the hydroxyl functional groups through hydrogen bonding. Filtration experiments with model foulants (bovine serum albumin (BSA) protein solution, humic acid solution (HA) and sodium alginate solution (SA)) were performed to estimate the antifouling properties of the newly developed nanofiltration membranes. As a result of surface modification proposed in this study the antifouling properties of an amphiphilic modified F-PA/PSF membrane were enhanced more than $10 \%$ compared to the PA/PSF specimen in terms of flux recovery ratio.

\section{Introduction}

Resource depletion and environmental pollution have become an exigent global issue that threatens daily life. Membrane technology becomes attractive in this context, as it is an energysaving and environmentally friendly technology. Nanofiltration membranes (NF), which have a high permeation flux under low operational pressures, have been applied in water purification, pretreatment for seawater desalination, removal of heavy metals, textile dyeing and other fields. ${ }^{1-3}$ Thin-film composite (TFC) membranes are the most popular commercial NF membranes which usually consist of an ultra-thin selective layer upon the surface of a porous flat sheet substrate membrane through the interfacial polymerization (IP) process, using diamine and acyl chlorides. ${ }^{4,5}$ However, membrane fouling is a bottleneck for the practical application of NF in water and

${ }^{a}$ Center for Membrane Separation and Water Science \& Technology, Ocean College, Zhejiang University of Technology, Hangzhou 310014, PR China. E-mail: shenjn@ zjut.edu.cn

${ }^{b}$ College of Chemical Engineering, Zhejiang University of Technology, Hangzhou 310014, PR China

${ }^{c}$ Rey Juan Carlos University, 28942, Fuenlabrada-Madrid, Spain

${ }^{d}$ Department of Chemical Engineering, KU Leuven, Celestijnenlaan 200F, B-3001 Leuven, Belgium wastewater treatment. Fouling usually causes a significant loss of productivity and increased operational cost. ${ }^{6}$ Foulants in the water (like colloid substances, bacteria, and nature organic materials) can unsuitably absorb and deposit onto the membrane surface or into the pores and pore walls and thus cause membrane fouling, ${ }^{7,8}$ which results in a flux decline during filtration operation and a shorter lifetime. Many researchers $^{9-11}$ have suggested that the dominant reason of membrane fouling is the intrinsic hydrophobicity of the membrane materials. Besides, the roughness and charge of the surface, feed solution chemistry and process conditions also have an important influence on the membrane fouling. ${ }^{8}$

The pores of NF membranes are very small $(\sim 1 \mathrm{~nm})$, so that fouling is mainly manifested as surface fouling. ${ }^{12}$ Currently, several surface modification methods like surface grafting, ${ }^{13}$ surface blending ${ }^{14}$ and surface coating ${ }^{15}$ have been used to improve the antifouling performance of NF membranes. Commonly, surface covalent grafting of NF membrane is performed by using residual acyl chloride on the active layer to react with other functional groups capable of reacting with them. Zhu et al. ${ }^{16}$ studied the structure and properties of TFC hollow fiber NF membranes grafted with poly (amidoamine) dendrimer (PAMAM). The hydrophilicity and water permeation flux increased with the PAMAM grafting, while the salt rejection 
was hardly affected. The effect of the PAMAM grafted TFC membrane on the removal of heavy metal ions like $\mathrm{Pb}^{2+}, \mathrm{Cu}^{2+}$, $\mathrm{Ni}^{2+}, \mathrm{Cd}^{2+}, \mathrm{Zn}^{2+}$ and $\mathrm{As}^{5+}$ was also significant, the rejection was over $99 \%$.

At present, antifouling membranes can be divided into several categories according to different criteria, on the basis of hydropathy property of membranes surfaces, the membranes can be classified into three categories: ${ }^{17}$ hydrophilic antifouling surfaces, hydrophobic self-cleaning surfaces and amphiphilic surfaces. Hydrophilic modification of NF membranes is one of the main ways to enhance the antifouling properties of a membrane. It is based on the fact that the organic matters in the water, which are hydrophobic in nature, are more likely to attach on a membrane surface because of hydrophobic interactions. The advanced hydrophilicity of membrane surface is beneficial to mitigate these molecular interactions. ${ }^{18}$ Furthermore, the hydrophilic membrane surface promotes the formation of a hydration layer, which restricts the pollutant adsorption or deposition on the membrane surface. ${ }^{19}$ Many hydrophilic materials have been used to prepare highly antifouling NF membranes, for example, poly(ethylene glycol) (PEG)-based materials, ${ }^{20,21}$ zwitterionic materials; ${ }^{22,23}$ among the most popular inorganic nanoparticles are titanium dioxide $\left(\mathrm{TiO}_{2}\right),{ }^{8,24}$ graphene oxide (GO), ${ }^{25,26}$ and multi-wall carbon nanotubes (MWCNTs). ${ }^{14,27,28}$ The strategy of hydrophilic modification represents a fouling-resistance defense mechanism. ${ }^{29-31}$ Unlike hydrophilic surfaces, hydrophobic membrane surfaces follow another mechanism: the fouling-release defense mechanism. ${ }^{32-34}$ This works through the development of a low surface energy layer barrier on the membrane surface using siliconebased $^{35,36}$ and fluorine-based materials, ${ }^{37,38}$ with a surface energy of about $22 \mathrm{~mJ} \mathrm{~m}^{-2}$ and $10-18 \mathrm{~mJ} \mathrm{~m}^{-2}$, respectively. Since the low surface energy characteristics of self-cleaning surfaces can make the intermolecular forces between pollutants and surfaces minimization, so that the pollutants are easily removed through low hydrodynamic shear force or simple mechanical cleaning, yielding an attractive "fouling-release" property. ${ }^{39}$

Although many attempts have been made to enhance the antifouling abilities of membrane surfaces, there are still significant problems to be addressed. With respect to hydrophilic modified membranes, the hydrophilic segments (like PEG) would be easily washed out of the matrix under the large drag force from the filtration pressure, because of their linear structures and low compatibility with the membrane matrix. ${ }^{\mathbf{4 0 , 4 1}}$ The surface of the membrane is still exposed to the risk of pollutant adsorption and deposition, thus reducing the flux. Therefore, the hydrophilic modification of NF membrane surfaces may is not a foolproof solution to solve the fouling effects. As for the hydrophobic modified film, one of the biggest problems is that the introduction of low surface energy materials may cause the water flux decline. Thus, the amphiphilic surfaces containing both hydrophilic and low surface energy domains would be good candidates for membrane modification. The presence of hydrophilic domains guaranties high water fluxes and low flux decline as result of hydraulic layer formation that mitigates the interaction between potential pollutants and the membrane surface. On the other hand, the hydrophobic anchoring groups are entangled with the hydrophobic membrane matrix because of the well compatibility, correlative hydrophilic segments can be stabilized in the membrane surface, thus weakens the interaction force between pollutants and membrane surface. ${ }^{\mathbf{4 0 4 2}}$ The amphiphilic modification of NF membrane may thus meet the antifouling requirements of a much broader variety of pollutants.

For the past few years, many researchers have paid attention to develop the amphiphilic surfaces on ultrafiltration membranes with multi-defense fouling mechanisms. ${ }^{\mathbf{4 3 4 4}}$ The common method is to fabricate an amphiphilic polymer and then blend it into the casting solution to prepare antifouling membranes by different methods, such as surface segregation, ${ }^{31,36}$ phase separation, ${ }^{41,45}$ dip-coating ${ }^{46}$ or phase inversion. ${ }^{40}$ However, due to the large size of the synthesized copolymer molecules and much smaller pore size of the NF membrane, the abovementioned methods are not suitable for the preparation of the amphiphilic antifouling NF membrane taking into account the possible blockage of membrane pores. Thus, a new way is required to prepare amphiphilic NF membrane surfaces. Zhang et al. ${ }^{17}$ fabricated an amphiphilic NF membrane by a two-step surface modification of a polyamide NF membrane, this amphiphilic NF membrane contains both hydrophilic domains and low surface energy domains, which can combine the fouling-resistance defense mechanism and the fouling-release defense mechanism. Triethylenetetramine with primary amine groups was firstly introduced to the membrane surface by reacting with unreacted acyl chloride on the surface of polyamide NF membrane. And then 2,2,3,4,4,4-hexafluorobutylmethacrylate was covalently grafted to the surface of the membrane by a Michael addition reaction with these primary amine groups. The antifouling experiments of BSA solution, HA solution and SA solution turned out that the flux recovery rate of the amphiphilic membrane was higher than of polyamide NF membrane, therefore possessed better antifouling property. However, this procedure is performed under harsh reaction conditions, which needs be carried out at a higher temperature, and the reaction time is relatively long. This will undoubtedly increase the energy consumption, and limit its industrial application. In this study, a simple and effective method is proposed avoiding the drawbacks mentioned above. The process involved in this approach can be performed at room temperature with the enhanced reaction kinetics.

An amphiphilic nanofiltration membrane with hydrophilic domains and low surface energy domains was developed through a two-step surface modification. First, the polyamide NF membrane was fabricated by interfacial polymerization of piperazine (PIP) and trimesoyl chloride (TMC). Then TEOA having abundant hydrophilic functional groups, is cost-effective, environment-friendly and easily-obtained. It can be grafted to the surface by reacting with the residual acyl chloride group of NF membrane, which makes the NF membranes more hydrophilic. Finally, the PFTS which is usually known as a low surface energy material was covalently grafted on hydroxyl functional groups through hydrogen bonding. Morphology of the prepared 
membranes were characterized using atomic force microscopy (AFM), scanning electron microscopy (SEM), etc. Separation and rejection properties were investigated by filtration experiments of salts and PEGs. Filtration experiments with model foulants (BSA protein solution, HA solution and SA solution) were performed to estimate the antifouling property of the prepared NF membranes.

\section{Materials and methods}

\subsection{Materials and chemicals}

A flat-sheet nonwoven-reinforced polysulfone (PSF) support membrane with average molecular weight cut-off (MWCO) of $80000 \mathrm{~g} \mathrm{~mol}^{-1}$ was supplied by Hangzhou Water Treatment Technology Development Center (Hangzhou, China). Piperazine (PIP, 99\%), $n$-hexane ( $\geq 97 \%)$, trimesoyl chloride (TMC), sodium dodecyl sulfate (SDS, analytically pure), triethanolamine (TEOA, 98\%), bovine serum albumin (BSA, analytically pure), sodium alginate (SA, analytically pure), humic acid (HA, analytically pure), $1 H, 1 H, 2 H, 2 H$-perfluorodecyltrichlorosilane (PFTS, 95\%) and polyethylene glycol (PEG) with different molecular weights $\left(M_{\mathrm{w}}=200,300,400,600,800\right.$ and $\left.1000 \mathrm{Da}\right)$ were purchased from Macklin (Shanghai, China). Alcohol, sodium sulfate $\left(\mathrm{Na}_{2} \mathrm{SO}_{4}\right)$, and sodium chloride $(\mathrm{NaCl})$ were purchased from Shanghai Lingfeng Chemical Reagent Co. Ltd, China. All reagents were used as received without further purification. Pure water used throughout in this work was derived from a Milli-Q system (Millipore, US).

\subsection{Membrane preparation}

Fig. 1 shows the preparation procedure of the amphiphilic NF membrane, which contained a two-step surface modification of NF membrane. The nascent polyamide NF membrane was fabricated by interfacial polymerization of PIP and TMC on the
PSF support membrane. The PSF support membrane which fixed by polyfluortetraethylene framework was firstly soaked into $0.5 \mathrm{wt} \%$ PIP aqueous solution containing $0.1 \mathrm{wt} \%$ SDS for $2 \mathrm{~min}$, and then the aqueous solution was poured out. The membrane surface was rinsed with pure water to remove the remaining liquid droplets, followed by air dried at room temperature. Subsequently, the PIP impregnated membrane surface was brimming with $0.1 \mathrm{wt} \%$ TMC organic solution ( $n$-hexane) for $1 \mathrm{~min}$, and then the organic solution was poured out. The membrane surface was rinsed thoroughly with $n$-hexane, heat treatment of $60{ }^{\circ} \mathrm{C}$ for $10 \mathrm{~min}$ was further carried out to make sure completion of the polymerization reaction. The resultant NF membrane was named PA/PSF membrane. On this basis, the amphiphilic membrane was prepared by a two-step modification.

Firstly, the surface of a newly prepared PA/PSF membrane was coated with TEOA ethanol solution for $2 \mathrm{~min}$ and washed thoroughly with ethanol to remove the remaining TEOA solution and then pure water to remove the residual ethanol. Membranes prepared with TEOA contents of $1,2,3,4$ and $5 \%\left(\mathrm{v}_{\text {твоА }} / \mathrm{v}_{\text {solution }}\right)$ are referred to as $\mathrm{H}_{1}$-PA/PSF, $\mathrm{H}_{2}$-PA/PSF, $\mathrm{H}_{3}$-PA/PSF, $\mathrm{H}_{4}$-PA/PSF and $\mathrm{H}_{5}-\mathrm{PA} / \mathrm{PSF}$, respectively. The resultant hydrophilic modified membranes were stored in pure water. Secondly, the surface of the hydrophilic modified membrane was immersed in PFTS alcohol solution with for $2 \mathrm{~min}$. The membrane surface was flashed with alcohol to remove the remaining PFTS solution and then pure water to remove the remaining ethanol, followed by drying at $80{ }^{\circ} \mathrm{C}$ for $10 \mathrm{~min}$. After heat treatment, the fabricated membrane (designated as the F-PA/PSF membrane) was stored in pure water for the next experiment.

\subsection{Membrane characterization}

The NF membrane was submerged in water for 24 hours prior to the determination of chemical composition analysis and

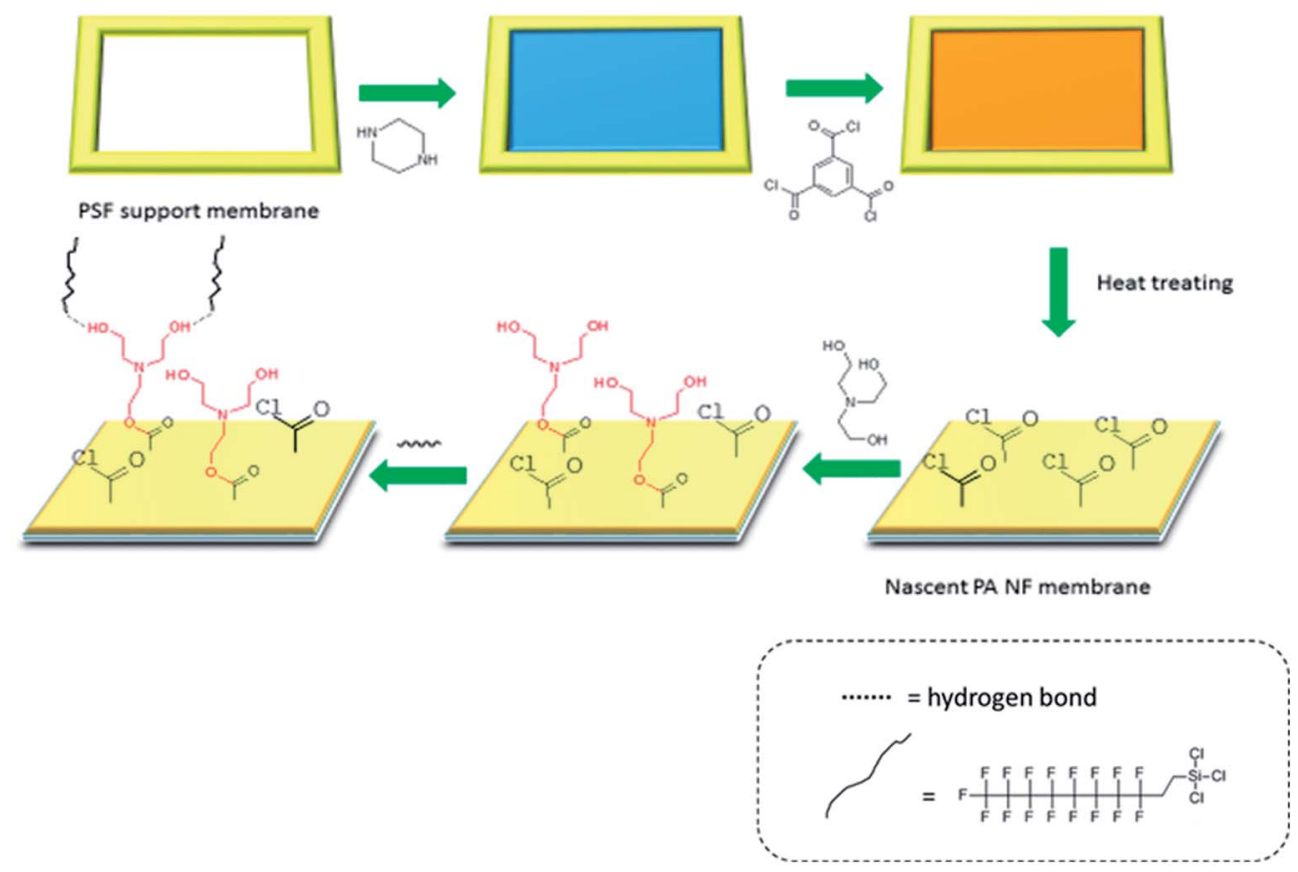

Fig. 1 Schematic diagram of amphiphilic NF membrane by a two-step modification. 
morphologies. Attenuated Total Reflectance-Fourier transform Infrared spectroscopy (ATR-FTIR, Nicolet 6700) and X-ray photoelectron spectroscopy (XPS, Kratos AXIS Ultra DLD, Japan) were used to characterize the chemical composition and structure of membrane surface before and after modification. The samples were scanned from 400 to $4000 \mathrm{~cm}^{-1}$ by ATR-FTIR with a resolution of $1 \mathrm{~cm}^{-1}$ for each spectrum. XPS were operated at constant pass energy of $100 \mathrm{eV}$ for wide scans and $20 \mathrm{eV}$ for detailed scans setting the O1s peak at BE $528.8 \mathrm{eV}$. The morphological characteristics of the membranes were observed with a scanning electron microscope (SEM, HITACHI, S4700A). The membrane samples were dried under vacuum and sputtercoated with gold for producing electric conductivity before observation. Atomic force microscopy (AFM, Bruker Dimension station) was used to characterize the surface topology of the membranes with a scanning area of $10 \mu \mathrm{m} \times 10 \mu \mathrm{m}$.

The water contact angle of pristine and modified membrane surfaces was measured using a contact angle goniometer (OCA20, Data-physics, Germany). For each membrane sample, at least five measurements were taken at different locations at room temperature and the average value was reported. The surface charge of the membranes were analyzed by an electrokinetic analyzer (SurPASS 3, Anton Paar, Austria) with $1 \mathrm{mM} \mathrm{KCl}$ as the electrolyte solution at a $\mathrm{pH}$ of 6.5-7.5 at room temperature. Zeta potential of the membrane was measured by an electrokinetic analyzer (SurPASS 3, Anton Paar, Austria) with $1 \mathrm{mM} \mathrm{KCl}$ as the electrolyte solution at a $\mathrm{pH}$ of 3.1-9.7 at room temperature.

\subsection{Membrane filtration testing}

The permeability and rejection tests were measured in a batch cross-flow NF membrane test unit at $25{ }^{\circ} \mathrm{C}$. All the membrane samples with effective area $19.6 \mathrm{~cm}^{2}(A)$ were pre-compacted at 1.1 MPa with pure water for $90 \mathrm{~min}$ to obtain a stable flux before testing and then evaluated for $30 \mathrm{~min}$ at a pressure of 1.0 MPa. The penetrating fluid is collected for the calculation of the water flux $(J)$ and salt rejection $(R)$ as follows:

$$
\begin{gathered}
J=\frac{V}{A t} \\
R(\%)=\left(1-\frac{C_{\mathrm{p}}}{C_{\mathrm{f}}}\right) \times 100
\end{gathered}
$$

where $t$ is the permeation time (h). $C_{\mathrm{p}}$ and $C_{\mathrm{f}}$ are the solute concentrations in permeate and feed, respectively. $1.0 \mathrm{~g} \mathrm{~L}^{-1}$ of $\mathrm{Na}_{2} \mathrm{SO}_{4}$ or $\mathrm{NaCl}$ solution was used to estimate the rejection performance of membrane. Molecular weight cut-off of membrane was tested using $1 \mathrm{~g} \mathrm{~L}^{-1}$ PEGs aqueous solution with different molecular weights $(200,300,400,600,800$, and 1000 Da). A total organic carbon analyzer (TOC Analyzer, Shimadzu TOC-VCNP) were used to determine the concentrations of the PEGs. The salt concentrations were measured by a conductivity meter (DDSJ-308A, Leizi, China), since the electric conductivity is proportional to the total salt concentration in a certain range. The average value of the three sets of parallel experimental data was used to calculate water flux and salt rejection.

\subsection{Antifouling performance of NF membranes}

Experiments with model foulants (BSA protein solution, HA and SA solution) were performed to estimate the antifouling property of NF membranes, which were chosen to represent protein, natural organic matter (NOM) and polysaccharide, respectively. The concentration of foulants solution was $1 \mathrm{~g} \mathrm{~L}^{-1}$. Before the antifouling experiment, the pure water flux $\left(J_{\mathrm{w} 1}\right)$ of $\mathrm{NF}$ membranes was measured at 1.0 MPa, and then the solution reservoir was filled with $1.0 \mathrm{~g} \mathrm{~L}^{-1}$ of foulant solution. Fouling tests were performed under the same pressure, the penetrating fluid was collected every $30 \mathrm{~min}$ for $24 \mathrm{~h}$, a steady flux $\left(J_{\mathrm{p}}\right)$ for foulant was obtained over a period of time. After that, the permeation returned to the feed tank to maintain a constant feed concentration. When the filtration of the foulants solution is finished, the membrane was cleaned with pure water for 30 minutes and the recovery flux after washing was measured $\left(J_{\mathrm{W} 2}\right)$. The flux recovery ratio (FRR) and total flux decline ratio $\left(\mathrm{DR}_{\mathrm{t}}\right)$ were used to signify the antifouling effect of NF membranes, defined as follows: ${ }^{47}$

$$
\begin{gathered}
\operatorname{FRR}(\%)=\left(\frac{J_{\mathrm{W} 2}}{J_{\mathrm{W} 1}}\right) \times 100 \\
\operatorname{DR}_{\mathrm{t}}(\%)=\left(\frac{J_{\mathrm{W} 1}-J_{\mathrm{p}}}{J_{\mathrm{W} 1}}\right) \times 100
\end{gathered}
$$

\section{Results and discussion}

\subsection{Characterization of NF membrane}

ATR-FTIR and XPS were used to understand the chemical structure of the skin layer of the NF membranes. Fig. 2 presents the ATR-FTIR spectra of the surfaces of PSF support membrane (a), nascent NF membrane PA/PSF (b), TEOA-grafted NF membrane $\mathrm{H}_{4}$-PA/PSF (c) and PFTS-grafted NF membrane F-PA/PSF (d). Compared with the spectrum of the PSF support membrane, an

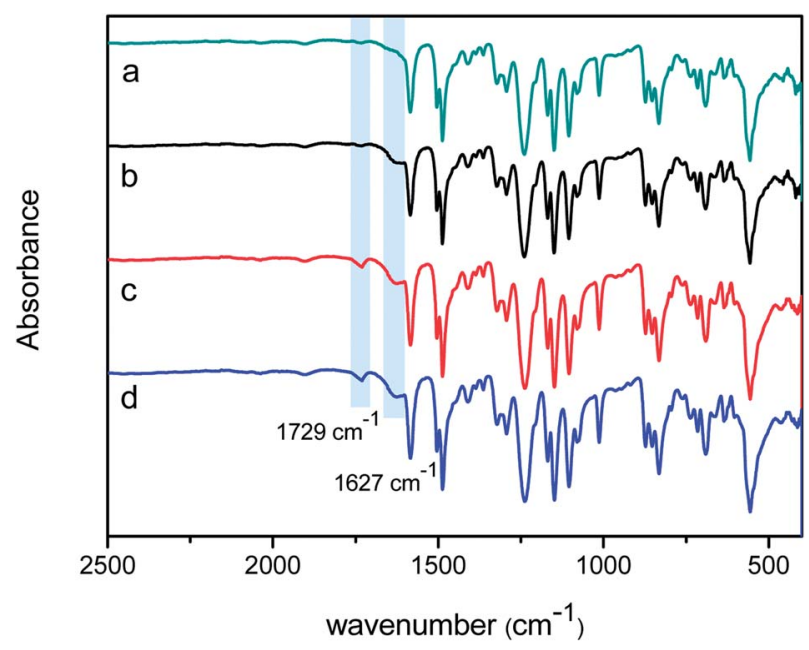

Fig. 2 ATR-FTIR spectra of the surfaces of (a) PSF membrane; (b) polyamide NF membrane PA/PSF; (c) TEOA-grafted NF membrane $\mathrm{H}_{4}{ }^{-}$ PA/PSF and (d) PFTS-grafted NF membrane F-PA/PSF. 
apparent absorption peak at $1627 \mathrm{~cm}^{-1}$ corresponding to $\mathrm{C}=\mathrm{O}$ stretching vibration of amide I appears in spectra (b), (c) and (d), confirming the formation of polyamide active layer after IP process. The new peak at $1729 \mathrm{~cm}^{-1}$ of membrane $\mathrm{H}_{4}$-PA/PSF (spectra (c)) is ascribed to $\nu_{\mathrm{C}=\mathrm{O}}$ of ester compound, which is formed by the esterification reaction between the hydroxyl groups of TEOA and the residual acyl chloride groups on the polyamide active layer. However, there is no difference between spectrum (c) and spectrum (d), this may be because the concentration of PFTS in membrane F-PA/PSF is too low to be detected by ATR-FTIR.

To make sure that the PFTS was successfully grafted onto the TEOA-modified membrane, an XPS characterization was further accomplished. Fig. 3 shows the XPS scans of PSF, PA/PSF, $\mathrm{H}_{4}-\mathrm{PA} /$ $\mathrm{PSF}$ and F-PA/PSF membrane. To distinguish the chemical changes of TEOA-modified NF membrane with different content of TEOA, O1s core-level spectra of membranes $\mathrm{H}_{1}-\mathrm{PA} / \mathrm{PSF}, \mathrm{H}_{2}-\mathrm{PA} /$ $\mathrm{PSF}, \mathrm{H}_{3}-\mathrm{PA} / \mathrm{PSF}, \mathrm{H}_{4}-\mathrm{PA} / \mathrm{PSF}$ and $\mathrm{H}_{5}-\mathrm{PA} / \mathrm{PSF}$ are shown in (b), (c), (d), (e) and (f). Compared with the PSF membrane, the appearance of N1s signal of PA/PSF membrane in (a) confirmed the formation of a polyamide active layer on the PSF membrane. The $\mathrm{O}$ contents of $\mathrm{H}_{4}$-PA/PES membrane is higher than of PA/PSF membrane, while the $\mathrm{N}$ and $\mathrm{C}$ contents were lower (O: $19.47 \% \rightarrow 22.13 \%$; N: $8.85 \% \rightarrow 7.73 \%$; C: $71.68 \% \rightarrow 70.34 \%$ ), as the grafted TEOA during first-step reaction. A new observed signal for F1s of F-PA/PSF membrane is an evidence that the PFTS has been successful grafted to the NF membrane surface. In the O1s core-level spectra of membranes, the peaks at approximately binding energy of $531.5,532.2$ and $533.3 \mathrm{eV}$ are attributed to the $\mathrm{N}-\mathrm{C}=\mathrm{O} * / \mathrm{O}-\mathrm{C}=\mathrm{O}^{*}, \mathrm{C}-\mathrm{O} *-\mathrm{H}$ and $\mathrm{O}=\mathrm{C}-\mathrm{O}^{*}$ functional groups, ${ }^{48}$ respectively. The $\mathrm{C}-\mathrm{O}^{*}-\mathrm{H}$ is introduced by TEOA, the conversion percentage (CP, \%) of carboxyl groups into ester bonds is calculated to quantitatively represent the grafting ratio of TEOA. The calculation formula of CP is as follows: ${ }^{49}$

$$
\mathrm{CP}=\left(1 / 2 A_{\mathrm{C}-\mathrm{O}^{*}-\mathrm{H}} / A_{\mathrm{O}=\mathrm{C}-\mathrm{O} *}\right) \times 100 \%
$$

where $A$ is the peak areas. The calculated $\mathrm{CP}$ of membranes $\mathrm{H}_{1}$ $\mathrm{PA} / \mathrm{PSF}, \mathrm{H}_{2}-\mathrm{PA} / \mathrm{PSF}, \mathrm{H}_{3}-\mathrm{PA} / \mathrm{PSF}, \mathrm{H}_{4}-\mathrm{PA} / \mathrm{PSF}$ and $\mathrm{H}_{5}-\mathrm{PA} / \mathrm{PSF}$ are $34.1 \%, 55.0 \%, 71.4 \%, 79.7 \%$ and $83.3 \%$, respectively.

The surface morphologies of the PSF and PA NF membranes were observed by SEM and AFM. As shown in Fig. 4, the PA/PSF membrane had a smooth surface (b) compared with the support PSF membrane (a). After the first-step of the modification, the $\mathrm{H}_{4}$ PA/PSF membrane surface appeared some small nodular structures (c), may be due to the aggregation of TEOA. After the second-step of the modification (d), the surface of the F-PA/PSF membrane was much rougher. AFM analysis was further carried out for quantitative investigation, and the results are shown in Fig. 4. It was found that the surface roughness of membranes slightly changed: the $R_{\mathrm{a}}$ values of membrane PA/PSF, $\mathrm{H}_{4}$-PA/PSF and F-PA/PSF were $7.7 \pm 0.3,10.4 \pm 1.4$, and $12.2 \pm$ 3.1 , respectively. A smooth surface restricts the adsorption and adhesion of pollutants, which is favorable to antifouling property. The results were in agreement with the SEM morphology observations.

As shown in Fig. 5, the hydrophilicity of the membrane surface was measured by the surface water contact angle (WCA).
The value of WCA decreased after the TEOA grafting, which means that the hydrophilicity of the membrane surface was increased. The enhancement of membrane hydrophilicity is caused by the increase of hydroxyl groups density in TEOA. This effect is beneficial for the water permeation as well as to mitigate the hydrophobic pollutant adsorption onto the membrane surface. This is conducive to the improvement of permeation flux and the reduction of fouling by hydrophobic substances. The membrane surface charge was evaluated through the zeta potential measurements at neutral $\mathrm{pH}$, depicted in Fig. 6 . Clearly that all the surface were negatively charged. The zeta potential of TEOA-modified membrane was less negatively charged in comparison to PA/PSF membrane. As increasing the TEOA content, the zeta potential negative charge decreases. This effect can be understood taking into account that some acyl chloride groups reacted with the hydroxyl functional groups of TEOA, thus the amount of the acyl chloride groups for hydrolysis decreased as result of increased amount of TEOA. ${ }^{48}$ The zeta potential of the membranes decreased after a two-step modification, which could be attributed to the introduction of HFBM. The substantial changes in the zeta potential of the membranes indicated the success of the chemical modification.

\subsection{Separation performance of membranes}

The preparation conditions during two-step surface modification (such as concentration of TEOA and PFTS) were examined to deduce the optimal separation performance of the modified NF membrane. The effect of the TEOA concentration on the permeation flux as well as on the $\mathrm{NaCl}$ and $\mathrm{Na}_{2} \mathrm{SO}_{4}$ rejection of the PA/PSF membranes during the first-step modification is displayed in Fig. 7. The water flux increased visibly when the TEOA concentration increased from 0 to $4 \%$, and then decreased when the TEOA concentration increased to 5\%. On the other hand, the $\mathrm{NaCl}$ and $\mathrm{Na}_{2} \mathrm{SO}_{4}$ rejection changes only slightly. The improved surface hydrophilicity of the TEOAmodified membranes is the main reason to the improvement of water flux. In addition, ethanol as a solvent facilitates the improvement of flux. This is because the polyamide layer and the PSF support membrane have different swelling degree in the ethanol solvent (swelling degree of the polyamide layer is larger than the support membrane), so the gap between the two layers increases in the ethanol solvent, which is favorable to the passage of more water molecules. ${ }^{17,50}$ The slight fluctuation in salt rejection is due to the lower electrostatic repulsive effect caused by the reduced surface negative charge. ${ }^{51}$ The effect of the PFTS concentration on the permeation flux as well as on the $\mathrm{NaCl}$ and $\mathrm{Na}_{2} \mathrm{SO}_{4}$ rejection of the PA/PSF membranes during second-step modification is presented in Fig. 8. The flux clearly declined after PFTS was grafted due to the hydrophobicity of PFTS. When the content of PFTS increases from $0.05 \%$ to $1.0 \%$, the flux decreases from $46.3 \pm 1.0 \mathrm{~L}\left(\mathrm{~m}^{2} \mathrm{~h}\right)^{-1}$ to $39.6 \pm 0.9 \mathrm{~L}\left(\mathrm{~m}^{2}\right.$ h) ${ }^{-1}$, while the rejection of $\mathrm{Na}_{2} \mathrm{SO}_{4}$ and $\mathrm{NaCl}$ remains constants about $80 \%$ and $30 \%$, respectively.

Molecular weight cut-off (MWCO) values of the membranes were also determined using PEGs as model solutes. Fig. 9 shows the rejection of $\mathrm{PA} / \mathrm{PES}, \mathrm{H}_{4}$-PA/PSF and F-PA/PSF membranes 

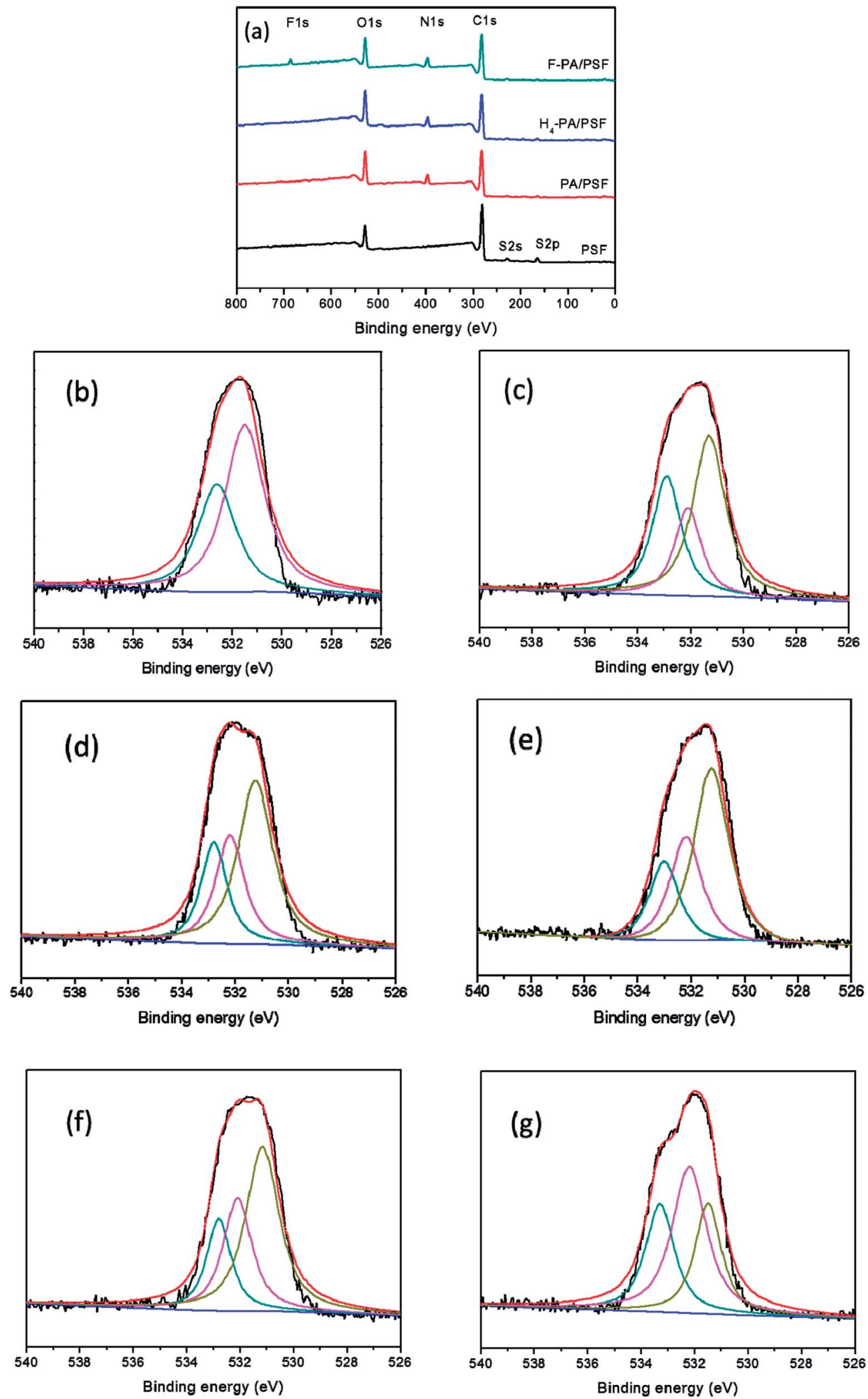

Fig. 3 XPS scans of membranes PSF, PA/PSF, $\mathrm{H}_{4}$-PA/PSF and F-PA/PSF membrane (a) and (b) O1s core level XPS spectra of membranes PA/PSF; (c) $\mathrm{H}_{1}$-PA/PSF; (d) $\mathrm{H}_{2}$-PA/PSF; (e) $\mathrm{H}_{3}$-PA/PSF; (f) $\mathrm{H}_{4}$-PA/PSF and (g) $\mathrm{H}_{5}$-PA/PSF.

for six PEGs solutions with different molecular weights (200, $300,400,600,800$, and $1000 \mathrm{Da})$. All tests were performed at 1.0 $\mathrm{MPa}$ and $25^{\circ} \mathrm{C}$. According to the solute rejection curve, the
MWCO of the PA/PSF, $\mathrm{H}_{4}$-PA/PSF and F-PA/PSF membranes were around $305 \mathrm{Da}, 290 \mathrm{Da}$ and $290 \mathrm{Da}$, respectively, indicating that the surface modification did not alter the pore size of 


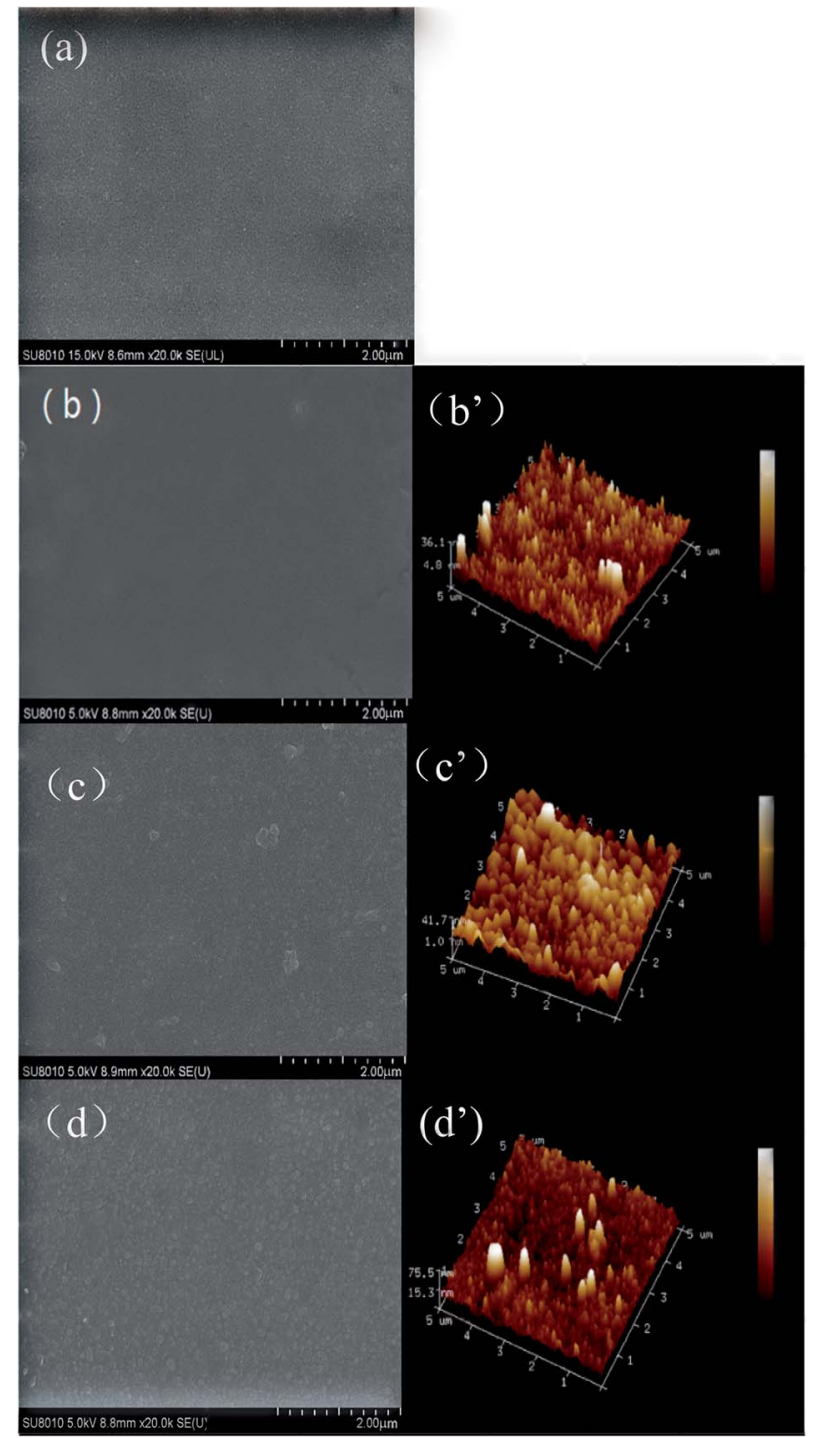

Fig. 4 The surface morphology of (a) PSF; (b) PA/PSF; (c) $\mathrm{H}_{4}-\mathrm{PA} / \mathrm{PSF}$; and (d) F-PA/PSF membranes; three-dimensional AFM images of $\left(b^{\prime}\right)$ PA/PSF; (c') $\mathrm{H}_{4}$-PA/PSF; and (d') F-PA/PSF membranes.

membranes. The rejection performance of modified membrane is hardly affected by the chemical modification proposed in this study ${ }^{48,52}$ This is consistent with the observed salt rejections.

\subsection{Antifouling property of membranes}

The time-dependent normalized flux $\left(J_{\mathrm{p}} / J_{\mathrm{w} 1}\right)$ profiles of the PA/ PSF, $\mathrm{H}_{4}$-PA/PSF and F-PA/PSF membranes during filtration of aqueous solutions containing different model foulants are shown in Fig. 10. It is apparent that the fluxes of all membranes exhibited an obvious decline when a foulant solution was filtered. After some time, stable fluxes were obtained for all membranes. There are some interactions between membrane surface and foulants. On the one hand, the foulants deposit onto the membrane surface under the driving pressure and cause serious membrane fouling and flux decline. On the other hand, the foulants deposited on the membrane surface are removed from the membrane surface under the near surface

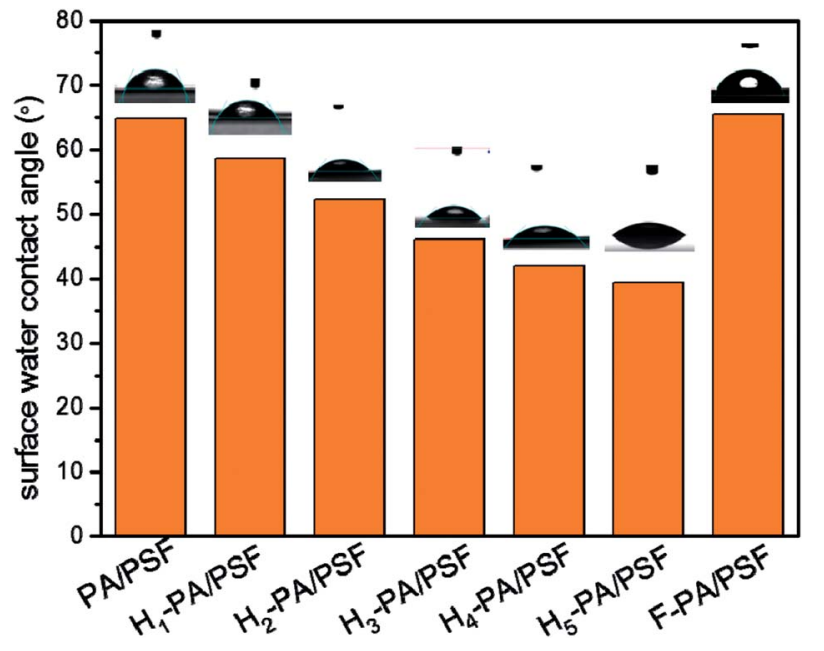

Fig. 5 Water contact angles of NF membranes. (The error in contact angle measurement was $<2^{\circ}$ ).

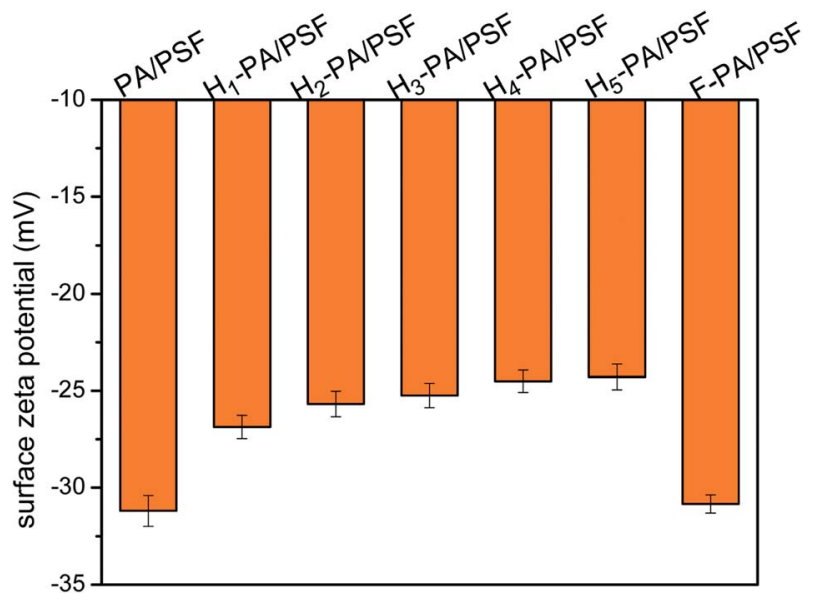

Fig. 6 Zeta potentials measurements for PA NF membranes $(\mathrm{pH}=7.0$ $\pm 0.2)$.

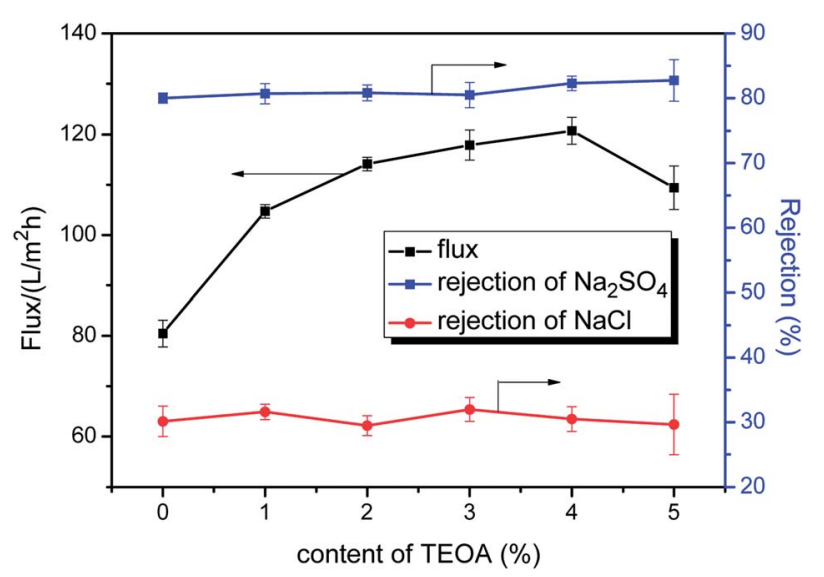

Fig. 7 Effect of the TEOA content on permeation flux and salt rejection of PA/PSF membranes during first-step modification (reaction time fixed at $2 \mathrm{~min}$, tested under $1.0 \mathrm{MPa}$ and $25^{\circ} \mathrm{C}$ ). 


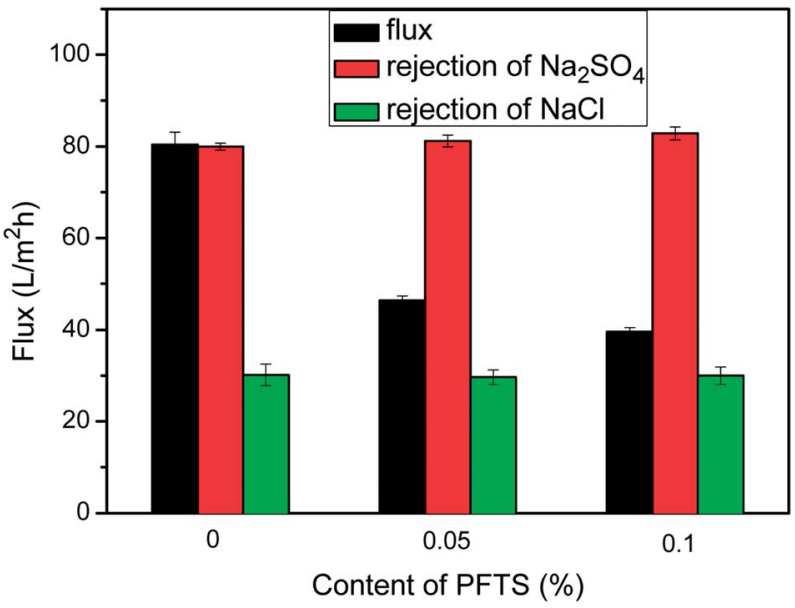

Fig. 8 Effect of PFTS content on permeation flux and salt rejection of PA/PSF membranes during second-step modification (reaction time $2 \mathrm{~min}$, reaction temperature $80^{\circ} \mathrm{C}$, testing was performed at $1.0 \mathrm{MPa}$ and $25^{\circ} \mathrm{C}$ ).

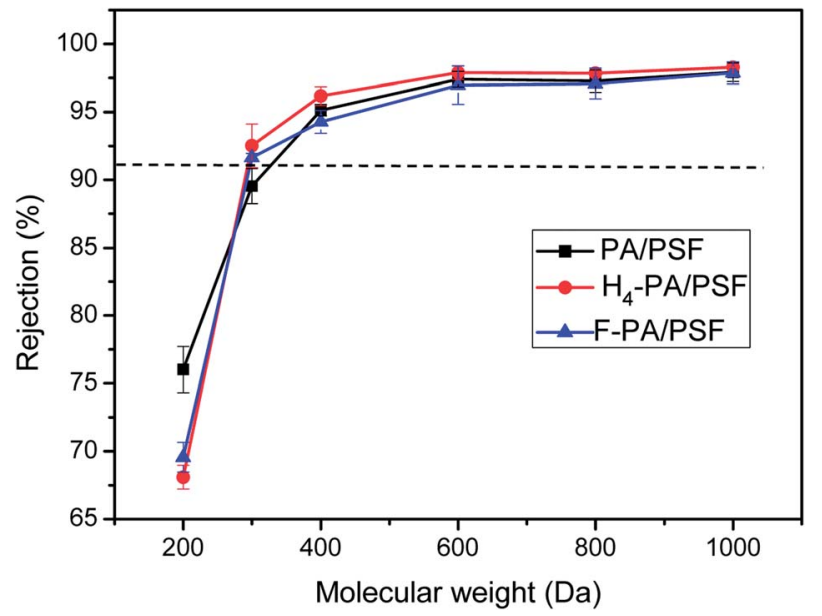

Fig. 9 Rejections to PEGS of PA/PSF, $\mathrm{H}_{4}-\mathrm{PA} / \mathrm{PSF}$ and $\mathrm{F}-\mathrm{PA} / \mathrm{PSF}$ membranes tested with $1 \mathrm{~g} \mathrm{~L}^{-1}$ aqueous solution at $1.0 \mathrm{MPa}$ and $25.0^{\circ} \mathrm{C}$.

shear stress. The flux tends to be stable when the adsorption and desorption of foulants reach to an equilibration under the joint effect of two kind of pressure. After this, a simple hydraulic cleaning was applied. The values $\left(\mathrm{FRR}, \mathrm{DR}_{\mathrm{t}}\right)$ calculated from fluxes before and after fouling and cleaning were used to evaluate the antifouling properties of the membranes. FRR and $\mathrm{DR}_{\mathrm{t}}$ values of $\mathrm{PA} / \mathrm{PSF}, \mathrm{H}_{4}$-PA/PSF and F-PA/PSF membranes for filtration of BSA, HA and SA aqueous solutions are given in Table 1. The FRR of the amphiphilic modified F-PA/PSF membrane is higher than that of the blank control PA/PSF membrane and the hydrophilic modified $\mathrm{H}_{4}-\mathrm{PA} / \mathrm{PSF}$ membrane, and the $\mathrm{DR}_{\mathrm{t}}$ is lower than that of the other two membranes during filtration of three different model foulant solutions. This manifests that the F-PA/PSF membrane has better antifouling properties. The good antifouling ability can be mainly ascribed to the amphiphilic nature of the F-PA/PSF membrane. On the one hand, the hydrophilic domains are
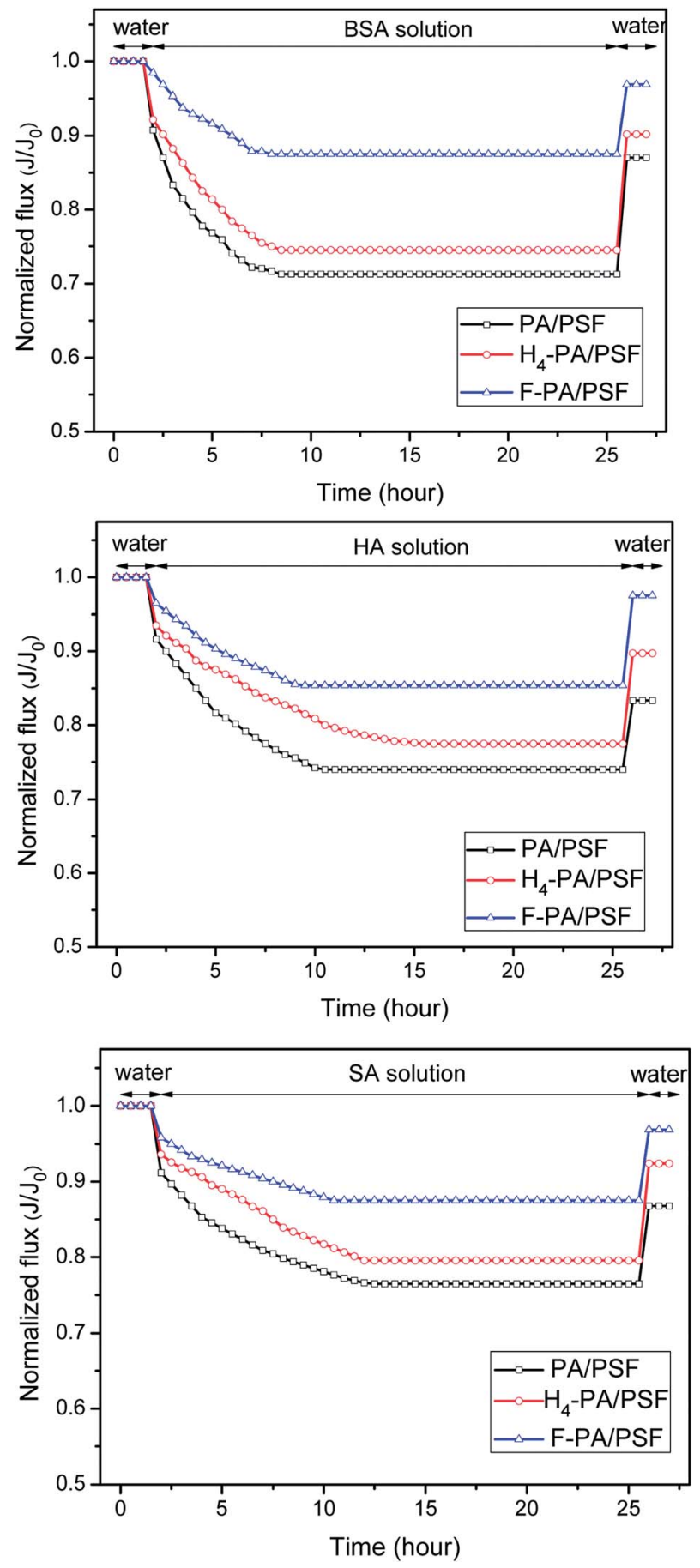

Fig. 10 Time-dependent normalized fluxes for PA/PSF, $\mathrm{H}_{4}-\mathrm{PA} / \mathrm{PSF}$ and F-PA/PES membranes during filtration of BSA solution; HA solution and SA solution, respectively, under the pressure of $1.0 \mathrm{MPa}$, temperature of $25.0^{\circ} \mathrm{C}$.

beneficial to bind water molecules and form a compact hydration layer to alleviate the interaction between the membrane surface and potential foulants (fouling-resistant defense mechanism). On the other hand, the foulants can be readily detached under the hydrodynamic shear forces in the presence of hydrophobic segments with low surface energy barriers (fouling-release defense mechanism). 
Table 1 Flux recovery ratio (FRR) and total flux decline ratio $\left(D R_{t}\right)$ values of the membranes in antifouling experiments of BSA, HA and SA aqueous solutions

\begin{tabular}{|c|c|c|c|c|c|c|}
\hline \multirow[b]{2}{*}{ Membrane } & \multicolumn{3}{|c|}{ FRR (\%) } & \multicolumn{3}{|c|}{$\mathrm{DR}_{\mathrm{t}}(\%)$} \\
\hline & BSA & HA & SA & BSA & HA & SA \\
\hline $\mathrm{PA} / \mathrm{PSF}$ & 87.0 & 83.3 & 86.8 & 28.7 & 26.0 & 23.5 \\
\hline $\mathrm{H}_{4}-\mathrm{PA} / \mathrm{PSF}$ & 90.2 & 89.8 & 92.4 & 25.4 & 22.5 & 20.43 \\
\hline F-PA/PSF & 96.9 & 97.5 & 96.8 & 12.5 & 14.6 & 12.5 \\
\hline
\end{tabular}

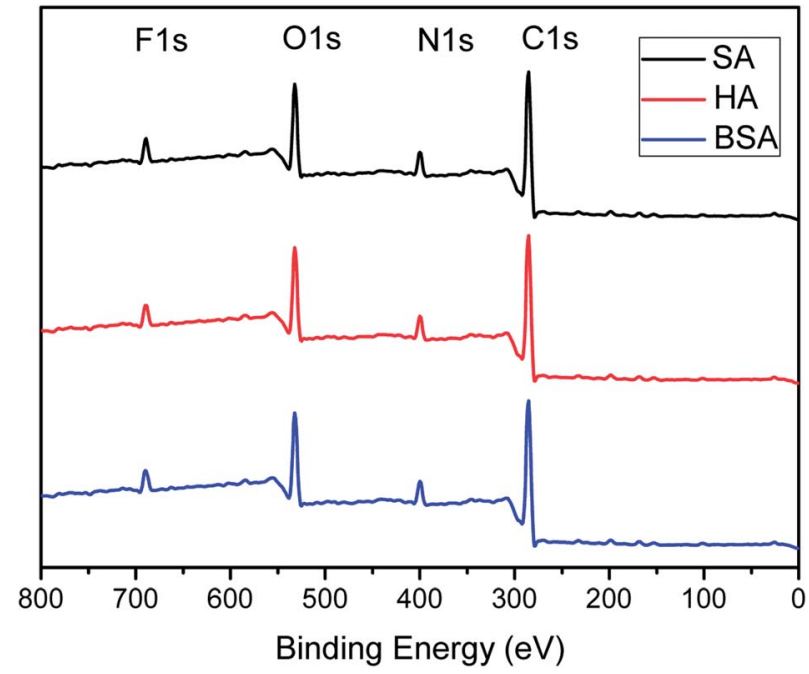

Fig. 11 XPS scans of membranes of F-PA/PSF membrane after foulants (BSA, HA, SA) filtration and hydraulic cleaning.

To make sure the PFTS was not washed out during the highpressure filtration testing or the hydraulic cleaning in the antifouling test. XPS characterizations of the filtrated and the fouled-cleaned F-PA/PSF membranes was further carried out to verify the existence of PFTS. The result was depicted in Fig. 11. Compared with the Fig. 3(a), the presence of F1s signal meant the stable existence of PFTS. So the amphiphilic modification of NF membrane was fairly stable under foulants filtration experiment and hydraulic cleaning.

\section{Conclusions}

In this study, a simple and effective method was employed to fabricate an amphiphilic NF membrane with hydrophilic domains and low surface energy domains through a two-step surface modification. The surface modification resulted in an unimportant decrease of the MWCO from 305 Da to $290 \mathrm{Da}$, while the $\mathrm{Na}_{2} \mathrm{SO}_{4}$ rejection increased slightly from $80.0 \% \pm 0.8$ (PA/PSF) and $82.3 \% \pm 1.1\left(\mathrm{H}_{4}\right.$-PA/PSF) to $82.9 \% \pm 1.3$, and maintaining a constant $\mathrm{NaCl}$ rejection of around $30 \%$. Compared with the control PA/PSF membrane and the hydrophilic modified $\mathrm{H}_{4}$-PA/PSF membrane, the antifouling properties of the amphiphilic modified F-PA/PSF membrane were clearly enhanced. FRR values were $96.9 \%$, 97.5\%, and $96.8 \%$ for a BSA solution, HA solution and SA solution, respectively. As expected, the two-step surface modification did not alter the pore size of membranes. The membrane after amphiphilic modification was fairly stable, and PFTS on the surface were not washed out throughout the experiment. In addition, it should be noted that the introduction of hydrophobic PFTS in F-PA/PSF membrane promotes the water flux decline. Hence, this investigation can provide a method for fabrication of antifouling $\mathrm{NF}$ membranes under mild conditions.

\section{Conflicts of interest}

There are no conflicts to declare.

\section{Acknowledgements}

The authors gratefully acknowledge the financial support from the National key research and development plan (No. 2017YFC0403700) and assistance to Qinghai Project of the Science and Technology Committee of Zhejiang Province (2018C26004).

\section{References}

1 A. Farsi, C. Malvache, O. D. Bartolis, G. Magnacca, P. K. Kristensen, M. L. Christensen and V. Boffa, Design and fabrication of silica-based nanofiltration membranes for water desalination and detoxification, Microporous Mesoporous Mater., 2017, 237, 117-126.

2 H. Li, W. Shi, W. Wang and H. Zhu, The extraction of sericin protein from silk reeling wastewater by hollow fiber nanofiltration membrane integrated process, Sep. Purif. Technol., 2015, 146, 342-350.

3 L. Ge, B. Wu, Q. Li, Y. Wang, D. Yu, L. Wu, J. Pan, J. Miao and T. Xu, Electrodialysis with nanofiltration membrane (EDNF) for high-efficiency cations fractionation, J. Membr. Sci., 2016, 498, 192-200.

4 B. W. Zhou, H. Z. Zhang, Z. L. Xu and Y. J. Tang, Interfacial polymerization on PES hollow fiber membranes using mixed diamines for nanofiltration removal of salts containing oxyanions and ferric ions, Desalination, 2016, 394, 176-184.

5 H. Li, W. Shi, Q. Du, R. Zhou, H. Zhang and X. Qin, Improved separation and antifouling properties of thin-film composite nanofiltration membrane by the incorporation of cGO, Appl. Surf. Sci., 2017, 407, 260-275.

6 N. Park, B. Kwon, I. S. Kim and J. Cho, Biofouling potential of various NF membranes with respect to bacteria and their soluble microbial products (SMP): Characterizations, flux decline, and transport parameters, J. Membr. Sci., 2005, 258, 43-54.

7 J. H. Jhaveri and Z. V. P. Murthy, A comprehensive review on anti-fouling nanocomposite membranes for pressure driven membrane separation processes, Desalination, 2016, 379, 137-154.

8 M. Safarpour, V. Vatanpour and A. Khataee, Preparation and characterization of graphene oxide/ $/ \mathrm{TiO}_{2}$ blended PES nanofiltration membrane with improved antifouling and separation performance, Desalination, 2016, 393, 65-78. 
9 H. Wang, X. Zhao and C. He, Innovative permeation and antifouling properties of PVDF ultrafiltration membrane with stepped hollow $\mathrm{SiO}_{2}$ microspheres in membrane matrix, Mater. Lett., 2016, 182, 376-379.

10 M. N. Z. Abidin, S. G. Pei, A. F. Ismail, M. H. D. Othman, H. Hasbullah, N. Said, S. H. S. A. Kadir, F. Kamal, M. S. Abdullah and B. C. Ng, Antifouling polyethersulfone hemodialysis membranes incorporated with poly (citric acid) polymerized multi-walled carbon nanotubes, Mater. Sci. Eng., C, 2016, 68, 540.

11 V. Vatanpour, M. E. Yekavalangi and M. Safarpour, Preparation and characterization of nanocomposite PVDF ultrafiltration membrane embedded with nanoporous SAPO-34 to improve permeability and antifouling performance, Sep. Purif. Technol., 2016, 163, 300-309.

12 P. Xu, J. E. Drewes, T. U. Kim, C. Bellona and G. Amy, Effect of membrane fouling on transport of organic contaminants in NF/RO membrane applications, J. Membr. Sci., 2006, 279, 165-175.

13 D. Y. Zhang, Q. Hao, J. Liu, Y. S. Shi, J. Zhu, L. Su and Y. Wang, Antifouling polyimide membrane with grafted silver nanoparticles and zwitterion, Sep. Purif. Technol., 2017, 192, 230-239.

14 M. R. Mahdavi, M. Delnavaz, V. Vatanpour and J. Farahbakhsh, Effect of blending polypyrrole coated multiwalled carbon nanotube on desalination performance and antifouling property of thin film nanocomposite nanofiltration membranes, Sep. Purif. Technol., 2017, 184, 119-127.

15 T. H. A. Ngo, D. T. Nguyen, K. D. Do, T. T. M. Nguyen, S. Mori and D. T. Tran, Surface modification of polyamide thin film composite membrane by coating of titanium dioxide nanoparticles, Journal of Science: Advanced Materials and Devices, 2016, 1(4), 468-475.

16 W. P. Zhu, J. Gao, S. P. Sun, S. Zhang and T. S. Chung, Poly(amidoamine) dendrimer (PAMAM) grafted on thin film composite (TFC) nanofiltration (NF) hollow fiber membranes for heavy metal removal, J. Membr. Sci., 2015, 487, 117-126.

17 R. Zhang, Y. Li, Y. Su, X. Zhao, Y. Liu, X. Fan, T. Ma and Z. Jiang, Engineering amphiphilic nanofiltration membrane surfaces with a multi-defense mechanism for improved antifouling performances, J. Mater. Chem. A, 2016, 4, 7892-7902.

18 X. Zhu, W. Tu, K. H. Wee and R. Bai, Effective and low fouling oil/water separation by a novel hollow fiber membrane with both hydrophilic and oleophobic surface properties, J. Membr. Sci., 2014, 466, 36-44.

19 A. Akbari, E. Aliyarizadeh, S. M. M. Rostami and M. Homayoonfal, Novel sulfonated polyamide thin-film composite nanofiltration membranes with improved water flux and anti-fouling properties, Desalination, 2016, 377, 11-22.

20 E. M. V. Wagner, A. C. Sagle, M. M. Sharma, Y. H. La and B. D. Freeman, Surface modification of commercial polyamide desalination membranes using poly(ethylene glycol) diglycidyl ether to enhance membrane fouling resistance, J. Membr. Sci., 2011, 367, 273-287.

21 X. You, T. Ma, Y. Su, H. Wu, M. Wu, H. Cai, G. Sun and Z. Jiang, Enhancing the permeation flux and antifouling performance of polyamide nanofiltration membrane by incorporation of PEG-POSS nanoparticles, J. Membr. Sci., 2017, 540, 454-463.

22 T. Ma, Y. Su, Y. Li, R. Zhang, Y. Liu, M. He, Y. Li, N. Dong, $\mathrm{H}$. $\mathrm{Wu}$ and $\mathrm{Z}$. Jiang, Fabrication of electro-neutral nanofiltration membranes at neutral $\mathrm{pH}$ with antifouling surface via interfacial polymerization from a novel zwitterionic amine monomer, J. Membr. Sci., 2016, 503, 101-109.

23 X. Weng, Y. Ji, F. Zhao, Q. An and C. Gao, Tailoring the structure of polyamide thin film composite membrane with zwitterions to achieve high water permeability and antifouling property, RSC Adv., 2015, 5, 98730-98739.

24 J. Wang, Y. Wang, J. Zhu, Y. Zhang, J. Liu and B. V. D. Bruggen, Construction of $\mathrm{TiO}_{2}$ @Graphene Oxide Incorporated Antifouling Nanofiltration Membrane with Elevated Filtration Performance, J. Membr. Sci., 2017, 533, 279-288.

25 M. Yang, C. Zhao, S. Zhang, P. Li and D. Hou, Preparation of graphene oxide modified poly(m-phenylene isophthalamide) nanofiltration membrane with improved water flux and antifouling property, Appl. Surf. Sci., 2016, 394, 149-159.

26 X. Li, C. Zhao, M. Yang, B. Yang, D. Hou and T. Wang, Reduced graphene oxide- $\mathrm{NH}_{2}$ modified low pressure nanofiltration composite hollow fiber membranes with improved water flux and antifouling capabilities, Appl. Surf. Sci., 2017, 419, 418-428.

27 G. Negin, S. S. Madaeni, D. Parisa, R. Hamid, S. Tahereh, R. Farshad and S. Bita, PES mixed matrix nanofiltration membrane embedded with polymer wrapped MWCNT: Fabrication and performance optimization in dye removal by RSM, J. Hazard. Mater., 2015, 298, 111-121.

28 J. Zheng, M. Li, K. Yu, J. Hu, X. Zhang and L. Wang, Sulfonated multiwall carbon nanotubes assisted thin-film nanocomposite membrane with enhanced water flux and anti-fouling property, J. Membr. Sci., 2017, 524, 344-353.

29 X. Zhao, Y. Su, H. Dai, Y. Li, R. Zhang and Z. Jiang, Coordination-enabled synergistic surface segregation for fabrication of multi-defense mechanism membranes, $J$. Mater. Chem. A, 2015, 3, 3325-3331.

30 G. He, Z. Jing, H. Shen, L. Li, Z. Li, Y. Li, L. Zhen, W. Hong, X. Yang and Z. Jiang, Functionalized Carbon Nanotube via Distillation Precipitation Polymerization and Its Application in Nafion-Based Composite Membranes, ACS Appl. Mater. Interfaces, 2014, 6, 15291.

31 Y. Liu, Y. Su, X. Zhao, R. Zhang, T. Ma, M. He and Z. Jiang, Enhanced membrane antifouling and separation performance by manipulating phase separation and surface segregation behaviors through incorporating versatile modifier, J. Membr. Sci., 2015, 499, 406-417.

$32 \mathrm{X}$. Zhu, H. E. Loo and R. Bai, A novel membrane showing both hydrophilic and oleophobic surface properties and its 
non-fouling performances for potential water treatment applications, J. Membr. Sci., 2013, 436, 47-56.

33 Y. Li, Y. Su, X. Zhao, R. Zhang, J. Zhao, X. Fan and Z. Jiang, Surface fluorination of polyamide nanofiltration membrane for enhanced antifouling property, J. Membr. Sci., 2014, 455, 15-23.

34 X. Zhao, W. Chen, Y. Su, W. Zhu, J. Peng, Z. Jiang, K. Lu, Y. Li and J. Liu, Hierarchically engineered membrane surfaces with superior antifouling and self-cleaning properties, $J$. Membr. Sci., 2013, 441, 93-101.

35 S. Sommer, A. Ekin, D. C. Webster, S. J. Stafslien, J. Daniels, L. J. Vanderwal, S. E. Thompson, M. E. Callow and J. A. Callow, A preliminary study on the properties and fouling-release performance of siloxane-polyurethane coatings prepared from poly(dimethylsiloxane) (PDMS) macromers, Biofouling, 2010, 26, 961-972.

36 X. Zhao, Y. Su, Y. Li, R. Zhang, J. Zhao and Z. Jiang, Engineering amphiphilic membrane surfaces based on PEO and PDMS segments for improved antifouling performances, J. Membr. Sci., 2014, 450, 111-123.

37 H. S. Sundaram, Y. Cho, M. D. Dimitriou, J. A. Finlay, G. Cone, S. Williams, D. Handlin, J. Gatto, M. E. Callow and J. A. Callow, Fluorinated amphiphilic polymers and their blends for fouling-release applications: the benefits of a triblock copolymer surface, ACS Appl. Mater. Interfaces, 2011, 3, 3366.

38 Z. Fei, H. Deng, X. Zhao, L. Xia, C. Yang, Y. Yang and A. Zhang, Fluorinated hyperbranched polyurethane electrospun nanofibrous membrane: Fluorine-enriching surface and superhydrophobic state with high adhesion to water, J. Colloid Interface Sci., 2014, 421, 49.

39 J. H. Jhaveri and Z. V. P. Murthy, Nanocomposite membranes, Desalin. Water Treat., 2015, 1-17.

40 F. Gao, G. Zhang, Q. Zhang, X. Zhan and F. Chen, Improved Antifouling Properties of Poly(Ether Sulfone) Membrane by Incorporating the Amphiphilic Comb Copolymer with Mixed Poly(Ethylene Glycol) and Poly(Dimethylsiloxane) Brushes, Ind. Eng. Chem. Res., 2015, 54, 8789-8800.

41 L. F. Fang, B. K. Zhu, L. P. Zhu, H. Matsuyama and S. Zhao, Structures and antifouling properties of polyvinyl chloride/ poly(methyl methacrylate)-graft-poly(ethylene glycol) blend membranes formed in different coagulation media, $J$. Membr. Sci., 2017, 524, 235-244.
42 Y. Liu, Y. Su, X. Zhao, Y. Li, R. Zhang and Z. Jiang, Improved antifouling properties of polyethersulfone membrane by blending the amphiphilic surface modifier with crosslinked hydrophobic segments, J. Membr. Sci., 2015, 486, 195-206.

43 J. A. Callow and M. E. Callow, Trends in the development of environmentally friendly fouling-resistant marine coatings, Nat. Commun., 2011, 2, 244.

44 Z. Zhao, H. Ni, Z. Han, T. Jiang, Y. Xu, X. Lu and P. Ye, Effect of surface compositional heterogeneities and microphase segregation of fluorinated amphiphilic copolymers on antifouling performance, ACS Appl. Mater. Interfaces, 2013, 5, 7808-7818.

45 J. Shen, Q. Zhang, Q. Yin, Z. Cui, W. Li and W. Xing, Fabrication and characterization of amphiphilic PVDF copolymer ultrafiltration membrane with high anti-fouling property, J. Membr. Sci., 2017, 521, 95-103.

46 Y. Chen, X. Zhao and C. He, Dual-mode antifouling ability of PVDF membrane with a surface-anchored amphiphilic polymer, RSC Adv., 2015, 5, 68998-69005.

47 W. Chen, Y. Su, J. Peng, Y. Dong, X. Zhao and Z. Jiang, Engineering a Robust, Versatile Amphiphilic Membrane Surface Through Forced Surface Segregation for Ultralow Flux-Decline, Adv. Funct. Mater., 2011, 21, 191-198.

48 F. Yan, H. Chen, L. Yang, Z. Lü, S. Yu, M. Liu and C. Gao, Improving the water permeability and antifouling property of thin-film composite polyamide nanofiltration membrane by modifying the active layer with triethanolamine, J. Membr. Sci., 2016, 513, 108-116.

49 J. Peng, Y. Su, W. Chen, X. Zhao, Z. Jiang, Y. Dong, Y. Zhang, J. Liu and X. Cao, Polyamide nanofiltration membrane with high separation performance prepared by EDC/NHS mediated interfacial polymerization, J. Membr. Sci., 2013, 427, 92-100.

50 A. Kulkarni, D. Mukherjee and W. N. Gill, Flux enhancement by hydrophilization of thin film composite reverse osmosis membranes, J. Membr. Sci., 1996, 114, 39-50.

$51 \mathrm{~J}$. Luo and Y. Wan, Effects of $\mathrm{pH}$ and salt on nanofiltrationa critical review, J. Membr. Sci., 2013, 438, 18-28.

52 J. Yin, Y. Yang, Z. Hu and B. Deng, Attachment of silver nanoparticles (AgNPs) onto thin-film composite (TFC) membranes through covalent bonding to reduce membrane biofouling, J. Membr. Sci., 2013, 441, 73-82. 